\title{
On the mechanism of extractive electrospray ionization (EESI) in the dual-spray configuration
}

\section{Journal Article}

\section{Author(s):}

Wang, Rui; Gröhn, Arto Juhani; Zhu, Liang; Dietiker, Rolf; Wegner, Karsten; Günther, Detlef; Zenobi, Renato

Publication date:

2012-03

Permanent link:

https://doi.org/10.3929/ethz-b-000161068

Rights / license:

In Copyright - Non-Commercial Use Permitted

Originally published in:

Analytical and Bioanalytical Chemistry 402(8), https://doi.org/10.1007/s00216-011-5471-8 


\title{
On the mechanism of extractive electrospray ionization (EESI) in the dual-spray configuration
}

\author{
Rui Wang • Arto Juhani Gröhn • Liang Zhu • \\ Rolf Dietiker • Karsten Wegner • Detlef Günther • \\ Renato Zenobi
}

Received: 29 July 2011 /Revised: 19 September 2011 /Accepted: 3 October 2011 / Published online: 16 October 2011

(C) Springer-Verlag 2011

\begin{abstract}
Dual-spray extractive electrospray ionization (EESI) mass spectrometry as a versatile analytical technique has attracted much interest due to its advantages over conventional electrospray ionization (ESI). The crucial difference between EESI and ESI is that in the EESI process, the analytes are introduced in nebulized form via a neutral spray and ionized by collisions with the charged droplets from an ESI source formed by spraying pure solvent. However, the mechanism of the droplet-droplet interactions in the EESI process is still not well understood. For example, it is unclear which type of droplet-droplet interaction is dominant: bounce, coalescence, disruption, or fragmentation? In this work, droplet-droplet interaction was investigated in detail based on a theoretical model. Phase Doppler anemometry (PDA) was employed to investigate the droplet behavior in the EESI plume and provide the experimental data (droplet size and velocity) necessary for theoretical analysis. Furthermore, numerical simulations were performed to clarify the influence of the sheath gas flow on the EESI process. No coalescence between the droplets in the ESI spray and the droplets in the sample spray was observed using various geometries and sample flow rates. Theoretical analysis, together with the PDA results, suggests that droplet fragmentation may be the dominant type of droplet-droplet interaction in the EESI. The interaction time between the ESI droplet and the sample
\end{abstract}

Published in the $A N A K O N$ special issue with guest editors P. Dittrich, D. Günther, G. Hopfgartner, and R. Zenobi.

R. Wang $\cdot$ L. Zhu $\cdot$ R. Dietiker $\cdot$ D. Günther $\cdot$ R. Zenobi $(\bowtie)$ Department of Chemistry and Applied Biosciences, ETH Zurich, 8093 Zurich, Switzerland

e-mail: zenobi@org.chem.ethz.ch

A. J. Gröhn · K. Wegner

Department of Mechanical and Process Engineering, ETH Zurich, 8092 Zurich, Switzerland droplet was estimated to be $<5 \mu$ s. This work gives a clear picture of droplet-droplet interactions in the dual-spray EESI process and detailed information for the optimization of this method for future applications that require higher sensitivity.

Keywords Mass spectrometry - Extractive electrospray . Ionization mechanisms

\section{Introduction}

Extractive electrospray ionization mass spectrometry (EESI-MS) in the dual-spray configuration, a technique derived from electrospray ionization (ESI) and first introduced by Cooks and coworkers in 2006 [1], has evolved into a powerful and versatile analytical method. Due to its advantages over ESI, it is widely applied in many fields requiring high-throughput analysis, such as online detection of chemical reaction products and intermediates [2], detection of native biomolecules [3], environmental monitoring in the field [4], and in vivo metabolomics [5]. This dual-spray configuration is also applied in other ambient ionization methods such as liquid sampling desorption ionization [6]. In the traditional ESI process, dissolved analytes are delivered through a capillary. With the help of a high electric field, and sometimes assisted with a sheath gas, a plume of charged droplets is formed. Gaseous ions are produced after several desolvation steps. The last step, the release of an unsolvated ion, can be described either by the ion evaporation model [7] or by the charged residue model [8]. ESI has a relatively low tolerance to the presence of buffers, salts, and complex matrices, which can lead to serious ion suppression effects. On the contrary, in the EESI process, the liquid sample is atomized using a sheath gas in the absence of an electric field and forms 
neutral droplets. These droplets are dispersed into a conventional ESI plume formed from pure solvent and collide with the charged ESI droplets in a Y-shaped intersection, where the analytes interact with the charged ESI droplets. Finally, in the merged plume, the analyte molecules mixing with the charged solvent in the droplets become gaseous ions in a subsequent ESI-like process. Because the analyte is dispersed over a large volume, EESI does not require any sample pretreatment. Another advantage is that the solvents in the charged ESI spray can be tuned to selectively extract the analytes, which are needed for the MS analysis, in complex matrices [9]. These advantages render EESI an ideal secondary ionization method for the analysis of gaseous volatile, semi-volatile, and even nonvolatile substances in various complex matrices such as exhaled breath [10, 11], milk [12, 13], and with viscous liquids [14-16].

Despite the wide scope of applications, there are still a lot of questions regarding the charging of analyte molecules. For instance, how exactly do the droplets from the ESI spray and sample spray interact with each other in the Y-shaped intersection? Does a total coalescence occur between the droplets? Should this not be the case, there are three other types of droplet-droplet collision in addition to total coalescence: bounce, disruption, and fragmentation (as illustrated in the inset of Fig. 1) [17, 18]. What is the dominant type of interaction between the droplets from the ESI spray and the sample spray? Furthermore, what is the mechanism for the corresponding charging process of analytes? A clear answer to these questions would definitely lead to a deeper understanding of the EESI process, which is important for optimizing the performance of the EESI method in terms of sensitivity, universality, and reproducibility. In our previous work using laser-induced fluorescence, it has been demonstrated that for nonvolatile compounds, the charged ESI droplets and the neutral sample droplets do collide in liquid form before the final desolvation and gas phase ion formation take place [9]. In addition, we found a strong dependence of ion signals on the analyte solubility in both the ESI and sample spray solvents, implying that a selective extraction occurs between the charged ESI droplets and the neutral sample droplets. This excludes total coalescence as the dominant type of droplet-droplet interaction between the ESI droplets and the neutral sample droplets with different solvents in the two sprays.

However, whether coalescence happens when the solvents in the ESI spray and sample spray are identical and the reasons for the presence or absence of total coalescence of the droplets in the EESI process are still unclear. To further optimize the performance of EESI, a detailed investigation was conducted based on a theoretical model to distinguish the type of droplet-droplet interaction in the EESI process. Concrete information about the droplet size and velocity is essential for theoretical analysis. However, mass spectrometric measurements cannot provide such information about the droplets in the EESI process [9]. As a complement to MS, we thus used phase Doppler anemometry (PDA) in this work. PDA is a powerful tool to directly measure the droplet size, velocity, and number density in dynamic processes such as electrospray [19-21] or desorption electrospray ionization [22]. The size, velocity, and density of droplets in the intersection where two sprays meet (referred to as the origin in the following) and $2 \mathrm{~mm}$ downstream from the cross-section were measured. Furthermore, the behavior of droplets in singlespray mode (defined in the "Experimental and methods") was also studied for comparison with results from EESI in order to understand how the droplet behavior changes when they collide with droplets from the second spray. In addition, the effect of experimental parameters such as the precise geometry and sample flow rate on droplet behavior
Fig. 1 Schematic diagram of the PDA measurements and the EESI setup. The inset shows four possible processes following droplet-droplet collision

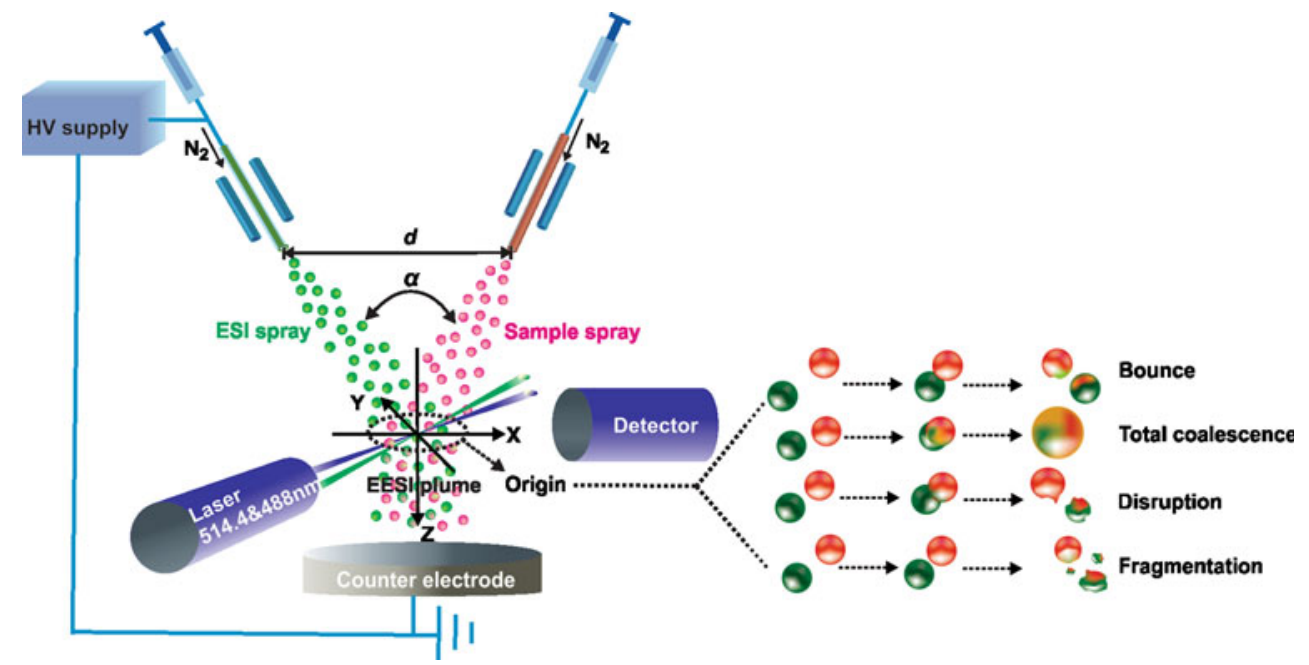


was investigated. In order to clarify the phenomena observed in the PDA measurements and to provide practical guidelines for optimizing EESI performance, numerical simulations of the gas flow dynamics in the intersection were conducted.

\section{Experimental and methods}

\section{PDA measurements}

The measurement scheme is shown in Fig. 1. The EESI setup consisted of two identical commercial electrospray sources (M955015DC6, Waters, Manchester, UK) which were constructed from two coaxial stainless capillaries for delivering the liquid and the sheath gas, respectively. The inner and outer diameters of these two capillaries are the same for both sources: 130/230 $\mu \mathrm{m}$ and 400/700 $\mu \mathrm{m}$, respectively. In the PDA measurements, one of the sources was used to spray the neutral sample, with a sheath gas pressure of 2 bar (compressed air); the other one was used as the ESI source, with a sheath gas (2 bar) and a voltage of $+4 \mathrm{kV}$ applied by a high-voltage supply (HCN140-12500, FuG, Rosenheim, Germany). The voltage was fixed for all the experiments. The solutions were delivered by two $500-\mu l$ syringes (Hamilton, Bonaduz, Switzerland) and a syringe pump (Harvard 22 syringe pump; Harvard Apparatus GmbH, March-Hugstetten, Germany) which provided a flow rate of $1-20 \mu \mathrm{l} / \mathrm{min}$. The distance between the tips of the capillaries and the counter electrode was $10 \mathrm{~cm}$. The measurements were performed with various geometries, with angles $(\alpha)$ of $30^{\circ}$, $80^{\circ}$, and $180^{\circ}$ and distances between the tips of the two capillaries $(d)$ of 3,4 , and $5 \mathrm{~mm}$, respectively.

A commercial phase Doppler anemometer (TSI Inc., St. Paul, MN, USA) was used to measure droplet velocity, droplet size, and droplet density in the EESI spray and single-spray modes (i.e., one spray was turned off and the other spray was kept on); the sheath gas was always on for both sprays. Two laser lines (488 and $514.5 \mathrm{~nm}$ ) from a water-cooled 5-W argon ion laser (LA-70-5, Innova 70, Coherent, Santa Clara, CA, USA) were used in the PDA setup. The laser beams illuminated the EESI spray, as shown in Fig. 1, and the scattered light from the droplets in the spray was collected by a fiber-optic-based receiver, which was $45^{\circ}$ off the axis of the incident beams, as depicted in Fig. 1. The ESI spray came from the negative $X$ direction, while the sample spray came from the positive $X$ direction; both flowed along the $Z$-axis after merging at the origin. The whole system was mounted on a threedimensional translation stage (9450-XYZ500, Isel Germany AG, Dermbach, Germany) controlled by a stepping motor (C142-4.1, Isel Germany AG) to allow moving the measurement point and making automated profile measurements. The
PDA measurement volume formed by the two intersecting laser beams had a half-axis of approximately $2.5 \mathrm{~mm}$ in length and $0.22 \mathrm{~mm}$ in width and height [23]. The measurements were done at 49 positions $(7 \times 7$ grid) in the $X Y$ plane by moving the measurement point automatically. The measurement area was defined according to the size of the spray plume. The detection ranges of droplet size, velocity, and density were $1-100 \mu \mathrm{m}, \approx 0-640 \mathrm{~m} / \mathrm{s}$, and $\geq 20 / \mathrm{cc}$, respectively [24]. Droplets with a size of $4 \mu \mathrm{m}$ only take $10 \mu$ s to be accelerated to follow the gas flow [25], a time short enough to consider the velocities of these droplets to be the same as the velocity of the sheath gas [26]. Thus, in this work, the gas velocity was derived from the velocities of droplets smaller than $4 \mu \mathrm{m}$ from the raw PDA data.

\section{Sample preparation}

In the EESI spray measurements, a water/ethanol mixture (1:1 volume ratio) was used in both sprays. Ethanol was bought from Merck (HPLC grade, Darmstadt, Germany); purified water (resistivity $\sim 18 \mathrm{M} \Omega \mathrm{cm}$ ) was obtained by using a water purification system (NANOpure, Barnstead, IA, USA).

\section{Numerical simulations}

Full three-dimensional numerical simulations were performed to calculate the pattern of the two sheath gas flows from the ESI and sample sprays using a computational fluid dynamics software package (ANSYS CFX 12.1, ANSYS, Berlin, Germany). The shear stress transport turbulence model was implemented in the simulations; when compared with the results of a pure laminar flow model at 2-bar backing pressure, no differences were found. The geometry of the numerical model was the same as that used in the PDA experiments, with an $80^{\circ}$ angle between the two sheath gas flows and a distance of $3 \mathrm{~mm}$ between the tips of the two spray capillaries. The dimensions of the inner and outer capillaries were identical to those in the experiments. The inlets of the two capillaries were in the same plane, and the inner capillary protruded $1 \mathrm{~mm}$ from the outlet of the outer capillary. The gas pressure at the inlets of the two capillaries was 2 bar, while the outlet pressure was set to open to the ambient pressure (i.e., 1 bar). The mesh size was set as fine as $3 \mu \mathrm{m}$ around the emitter to simulate the pattern precisely.

\section{Results and discussion}

Comparison of droplet sizes in EESI and in single-spray mode

With both the ESI and sample sprays on, the size, velocity, and density of droplets were measured simultaneously in 
the EESI spray plume under various experimental conditions using PDA. The normalized volume density vs. droplet size under various geometries is shown in Fig. 2. The Sauter mean diameter (SMD, the total droplet volume divided by the total droplet surface area) is usually used to characterize the droplets in the spray. The volume density rather than the number density was used to avoid bias from the large number of small droplets. The volume density was normalized to the sum of all droplet volumes in the measurement area in order to avoid an influence of a varying volume density in different measurements. In the case of $\alpha=80^{\circ}$, the SMD of the EESI spray was measured to be $7.85 \mu \mathrm{m}$ and the average SMD was calculated to be $8.82 \mu \mathrm{m}$ by averaging the results of the ESI spray and the sample spray in the single-spray mode, i.e., quite similar. If permanent total coalescence happens between the droplets from the two sprays, the droplet size distribution of the EESI spray should shift to a significantly larger value and the density of small droplets should decrease compared with the averaged data from the ESI spray and the sample spray in the single-spray mode. However, no obvious shift of the distributions was observed, i.e., there was no significant change of the droplet size or dramatic decrease of the droplet density after two sprays met, implying that no permanent coalescence takes place at the origin of the EESI plume $(z=0)$ even when the solvents in the two sprays are identical. This is fully in line with the results from our previous work [9] where different solvents were used for the two sprays. The droplet behavior $2 \mathrm{~mm}$ axially downstream from the origin was also measured, as shown in Fig. 2b. The droplet sizes for both sprays have decreased further compared with the ones at the origin due to the solvent evaporation; the decrease is stronger for the droplets in the sample spray. At $2 \mathrm{~mm}$, the SMD of the EESI spray $(6.65 \mu \mathrm{m})$ was similar to the average $(6.00 \mu \mathrm{m})$, and the droplet size distributions of the EESI spray and the combination of the two single sprays were analogous, suggesting again that there is no total coalescence of droplets $2 \mathrm{~mm}$ downstream.

Similar results were obtained when other EESI geometries were applied. In Fig. 2c, the droplet size distribution for $\alpha=30^{\circ}$ was comparable to the results for $\alpha=80^{\circ}$. Only in an extreme case $\left(\alpha=180^{\circ}\right.$, i.e., both sprays facing each other) did the droplet size in the ESI spray increase dramatically compared with other geometries, which may be caused by the particularly intense interactions between the droplets from two sprays in this case. The SMD of the EESI spray was $12.54 \mu \mathrm{m}$, while the average SMD was $14.07 \mu \mathrm{m}$. The droplet size distribution of the EESI spray assumed a slightly smaller value compared with the averaged results for the ESI spray and the sample spray in the single-spray mode. This reduction of the droplet size is probably a result of the breakup of droplets due to the large momentum produced by the collision between the droplets from the two sprays in this geometry. All results with different geometries suggest that there was no coalescence of the droplets when the ESI spray met with the sample spray at the origin. Furthermore, the droplet behavior with various sample flow rates for $\alpha=80^{\circ}$ was studied at the origin. The SMD of the EESI spray was similar to the average value at different sample flow rates, again suggesting the absence of coalescence. Both the sheath gas and the electric field influence the size of ESI droplets. On the contrary, only the sheath gas affects the size of the sample
Fig. 2 Normalized droplet volume density vs. droplet size in the EESI spray and single-spray mode obtained by the PDA measurements. a $\alpha=80^{\circ}, z=0 \mathrm{~mm}$ (at the origin). $\mathbf{b} \alpha=80^{\circ}$, $z=2 \mathrm{~mm}(2 \mathrm{~mm}$ downstream from the origin). $\mathbf{c} \alpha=30^{\circ}$, $z=0 \mathrm{~mm}$. $\mathbf{d} \alpha=180^{\circ}, z=0 \mathrm{~mm}$. The average is calculated and normalized from the results of the ESI and sample sprays in the single-spray mode
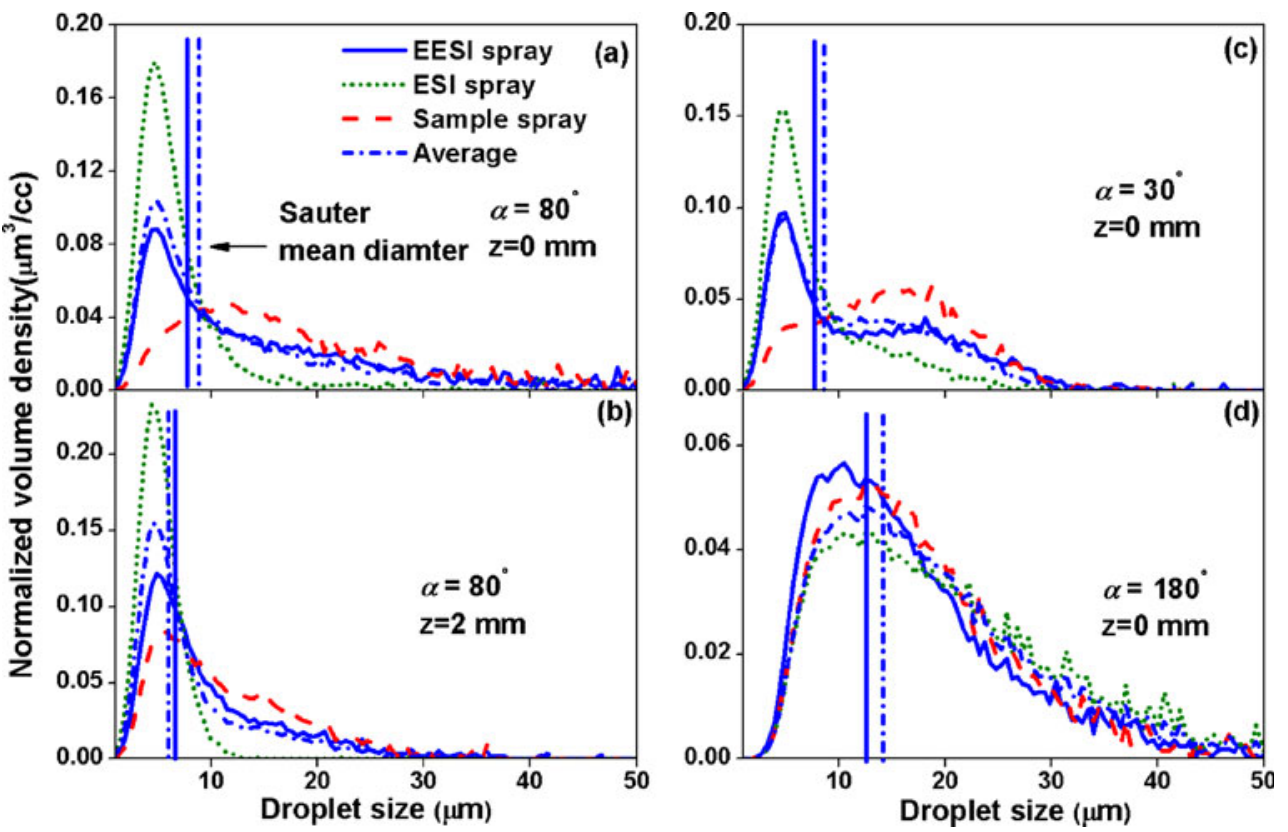
droplets. As a result, ESI droplets are normally smaller than the droplets from the neutral sample spray, as observed at low sample flow rates $(\leq 5 \mu \mathrm{l} / \mathrm{min})$. With a higher flow rate of $20 \mu \mathrm{l} / \mathrm{min}$, the size of the sample droplets shows no distinct change, while the size of the ESI droplets increases. Because the initial droplet size rises and the electric field was not high enough to break the large droplets into small ones, this caused a shift of the droplet size distribution of the ESI spray toward a higher value.

Investigation of droplet behavior at the origin of the EESI plume

In order to obtain more information on the droplets in EESI for the theoretical analysis, the droplet behavior was investigated in detail at the origin of the EESI plume. Contour plots of gas velocity, droplet velocity, normalized droplet volume density, and droplet size in the $X Y$ plane at $z=0$ are depicted for $\alpha=80^{\circ}$ (Fig. 3). The gas velocity profile of the EESI spray in Fig. 3a exhibits a nearly circular shape, implying a good fusion of the two sheath gas flows from the ESI spray and the sample spray after encountering each other at the origin. However, the direction of the profile was $45^{\circ}$ off the $Y$-axis to the left, which will be explained below. The highest gas velocity, $90 \mathrm{~m} / \mathrm{s}$, was at the center. The speed decreased gradually when going off-center, on account of the expanding gas flow. The gas velocity decreased to $60 \mathrm{~m} / \mathrm{s} 2 \mathrm{~mm}$ downstream from the origin of the EESI plume (Fig. 4a). When comparing the droplet velocity profiles (Fig. 3g-i) and the gas velocity profiles (Fig. $3 \mathrm{a}-\mathrm{c}$ ) in both the EESI and the single-spray modes, it becomes clear that the fast droplets were mostly located in the area of high-speed gas flow in both the EESI and the single-spray mode. At the origin, most droplets in the ESI spray were distributed roughly in two areas surrounding the conjunct gas flows (Fig. 3e), while the droplets in the sample spray were mostly distributed in one area beside the gas flows (Fig. 3f). The droplet distribution in the EESI spray was close to the sum of the results of the two-spray mode (Fig. 3d). In addition, the overlap area between the droplet distribution of the ESI spray and the distribution of the sample spray at the origin was fairly small. It is similar to the overlap area $2 \mathrm{~mm}$ from the origin (Fig. 4e, f). The small overlap area results in little chance for the interactions between the droplets from the two sprays.

At the origin, the size of most droplets in the ESI spray was approximately $3-5 \mu \mathrm{m}$ based on the contour figures of the droplet volume density distribution (Fig. 3e) and the droplet size distribution (Fig. 3k). This is consistent with the droplet size in the conventional ESI spray, which usually has a fairly narrow size distribution if submicronsized offspring droplets generated from Coulomb explosions are ignored [20]. The latter cannot be "seen" by PDA,

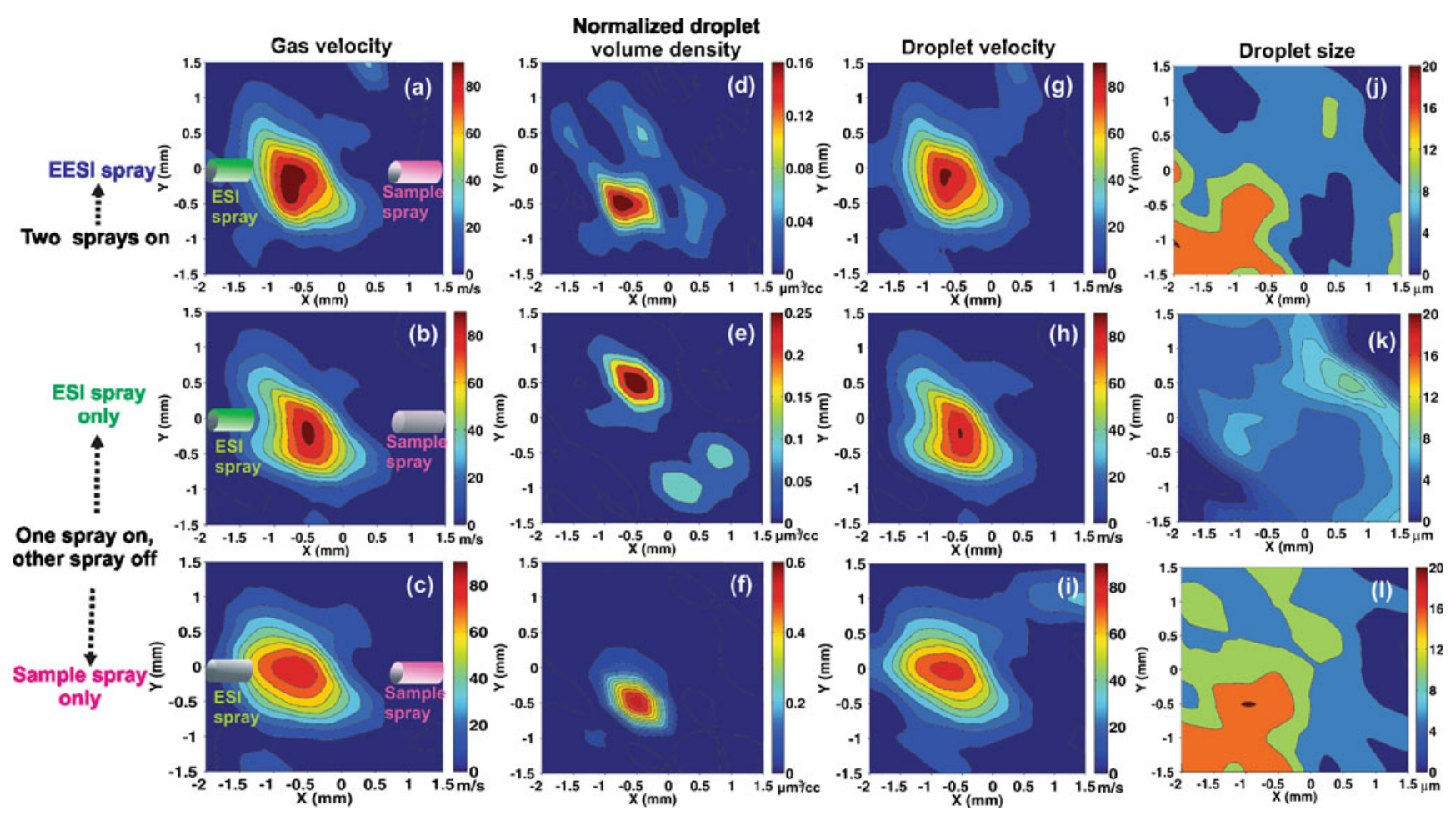

Fig. 3 Contour plots of the gas velocity $(\mathbf{a}-\mathbf{c})$, droplet volume density $(\mathbf{d}-\mathbf{f})$, droplet velocity $(\mathbf{g}-\mathbf{i})$, and droplet size $(\mathbf{j}-\mathbf{l})$ for the EESI spray and the two single-spray modes measured by PDA with $\alpha=80^{\circ}$ at the origin of the EESI plume 

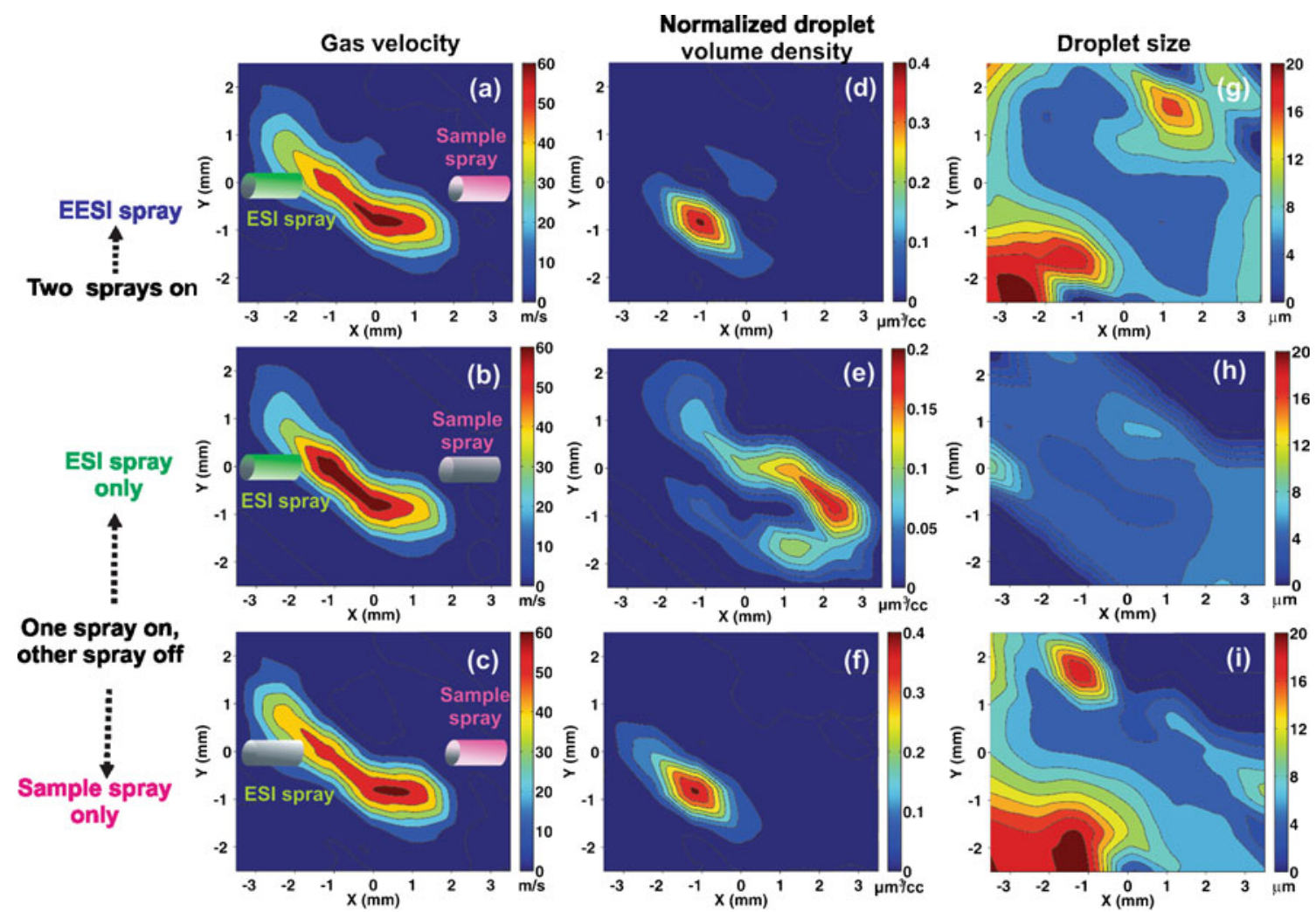

Fig. 4 Contour plots of the gas velocity $(\mathbf{a}-\mathbf{c})$, droplet volume density $(\mathbf{d}-\mathbf{f})$, and droplet size $(\mathbf{g}-\mathbf{i})$ for the EESI spray and the two single-spray modes measured by PDA with $\alpha=80^{\circ}$ and $2 \mathrm{~mm}$ from the origin of the EESI plume

which only can observe droplets larger than $\approx 1 \mu \mathrm{m}$. Compared with the volume density profile (Fig. 3f) and the droplet size distribution (Fig. 3i) of the sample spray, larger droplets were distributed at the place with higher droplet concentration, considered as the spray center. This is similar to the typical size distribution for a coaxial airassisted spray where the droplets are larger at the center along the radial direction of the spray [27]. The droplet size distribution in the EESI spray was approximately the average of the distributions of the two sprays in the single-spray mode. At $2 \mathrm{~mm}$ from the origin, the droplet size distributions in both the EESI and single-spray modes were similar to the distributions at the origin.

In order to better understand the effect of the gas flow on the EESI process, the pattern of two gas flows from the ESI spray and the sample spray was also investigated by numerical simulations. Droplets were not explicitly accounted for due to limitations in computing power. The velocity vector profiles of the gas flow pattern in the $X Y$ plane at the origin are shown in Fig. 5. When two sprays were in a perfect head-on position, the two gas flows converged and expanded along the $Y$-axis, $90^{\circ}$ off the spray direction (the $X$-axis; Fig. 5a). However, when the centerlines of two sprays were misaligned, e.g., with a $0.2-\mathrm{mm}$ displacement between the tips of two spray capillaries, the two gas flows still converged, but the conjunct gas flow was roughly $30^{\circ}$ off the $Y$-axis, as indicated in Fig. 5b. If the displacement increased to $0.4 \mathrm{~mm}$, the conjunct gas flow was roughly $45^{\circ}$ off the $Y$-axis, which is consistent with the experimental observations in the gas velocity profile of the EESI spray: the direction of the gas flow profile in the EESI spray was roughly $45^{\circ}$ off the $Y$-axis in the PDA measurements (Fig. 3a). This suggests that there was indeed a displacement of $\sim 0.4 \mathrm{~mm}$ between the tips of two spray capillaries, which is reasonable in the experimental scenario. It is nearly impossible to set up two sprays in an ideal head-on position in practice. The maximum velocity of the gas flow was $100 \mathrm{~m} / \mathrm{s}$, which is close to the experimental result of $90 \mathrm{~m} / \mathrm{s}$. Furthermore, the shape of the gas flow pattern in the simulations is similar to the shape of the gas velocity profile of the EESI spray measured by PDA.

The simulated velocity profiles of the two gas flows in the XZ plane, for $\alpha=80^{\circ}$ and a $0.4-\mathrm{mm}$ displacement between the tips of two spray capillaries, are shown in Fig. 6a. The $X Y$ profiles of these two gas flows downstream of the origin are also depicted in Fig. 6b. The gas velocity reduced to $70 \mathrm{~m} / \mathrm{s}$ at $2 \mathrm{~mm}$, which is similar to the gas velocity of $60 \mathrm{~m} / \mathrm{s}$ observed in the PDA measurements. The phenomena observed in the droplet volume density profiles 


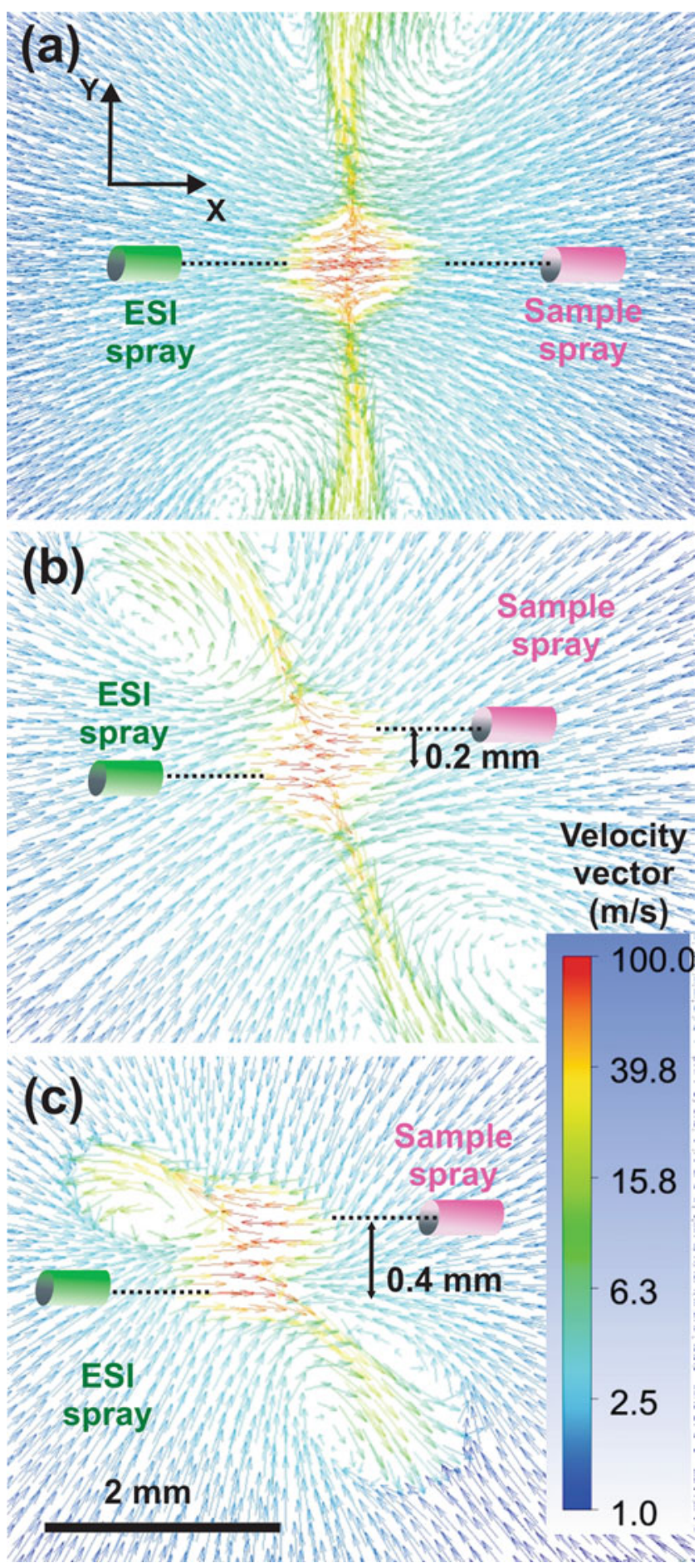

Fig. 5 Simulated velocity vector distribution of two gas flows in the $X Y$ plane at $z=0$ in the head-on position (a) and with the sprays displaced by $0.2 \mathrm{~mm}$ (b) and $0.4 \mathrm{~mm}$ (c). The geometry is $\alpha=80^{\circ}$. The color bar is scaled logarithmically

in single-spray mode at the origin (Fig. 3e, f) and $2 \mathrm{~mm}$ downstream (Fig. 4e, f) are then well represented by the simulations. At the origin, the two gas flows mix partially even when the two spray capillaries are not perfectly aligned; part of the gas expands in the opposite directions (yellowish lobes in Fig. 6b, top panel). Small droplets, such as the ones produced by the ESI spray, can more easily follow both expansion directions downstream. At $2 \mathrm{~mm}$, there is almost no overlap between the two gas flows. Droplets from the ESI spray seem to preferentially follow the ESI sheath gas rather than both sheath gas flows in two directions (Fig. 6b, bottom panel), although there is still some density in the sample spray direction (Fig. 4e). This effect is less pronounced for the sample spray droplets because of their larger size (Figs. 3f and 4f).

Theoretical analysis of droplet-droplet collision in the EESI process

There are four types of droplet-droplet collisions. (1) Bounce: there is a thin intervening gas film between the surfaces of two droplets. If the collision kinetic energy (CKE) of the two droplets is not sufficient to penetrate this gas layer, then the droplets bounce off each other, meaning that there is no physical contact between two liquid droplets and they only flatten temporarily. (2) Total coalescence: when the CKE of two droplets is high enough so that the thickness of the gas film reaches a critical value, usually $10 \mathrm{~nm}$ [28], two droplets will coalesce temporarily or permanently, depending on the CKE. If the CKE is not too high, the coalesced droplet will oscillate with an amplitude of a few nanometers and finally achieve a stable form. If the CKE is too high, there are two possibilities for droplet-droplet collision. (3) Disruption: two droplets temporarily coalesce/contact and afterwards separate into two droplets. (4) Fragmentation: after temporary coalescence/contact, the droplet undergoes catastrophic breakup into numerous satellite droplets [17].

Based on the results of the PDA measurements, it is clear that there is no permanent coalescence at the origin of the EESI plume because the droplet size in the EESI spray did not increase compared with the size in the single-spray mode. However, it is hard to decide which of the other three types is predominant in the EESI process by only relying on the PDA measurements. Here, the type of droplet collision in the EESI process is evaluated theoretically. A diagram of a droplet-droplet collision is shown in Fig. 7. One usually uses the impact parameter $(b)$ and the Weber number $(\mathrm{We})$ to determine the type of droplet collision instead of the CKE [17]. The impact parameter $(b)$ is the distance from the center of one droplet to the relative velocity vector $(U)$ placed on the center of the other droplet, as depicted in Fig. 6. $U$ is given by:

$U^{2}=V_{E}^{2}+V_{S}^{2}-2 V_{E} V_{S} \cos \theta$

where $V_{\mathrm{E}}$ and $V_{\mathrm{S}}$ represent the velocities of the ESI droplet and the sample droplet, respectively. $\theta$ is the angle between 
Fig. 6 a Simulated velocity profile of the two gas flows in the $X Z$ plane. b Gas velocity vector profiles of two gas flows in the $X Y$ plane downstream along the plume at the origin, $0.5 \mathrm{~mm}$ from the origin, and $2 \mathrm{~mm}$ from the origin, respectively. The geometry is $\alpha=80^{\circ}$. The color bars are scaled logarithmically

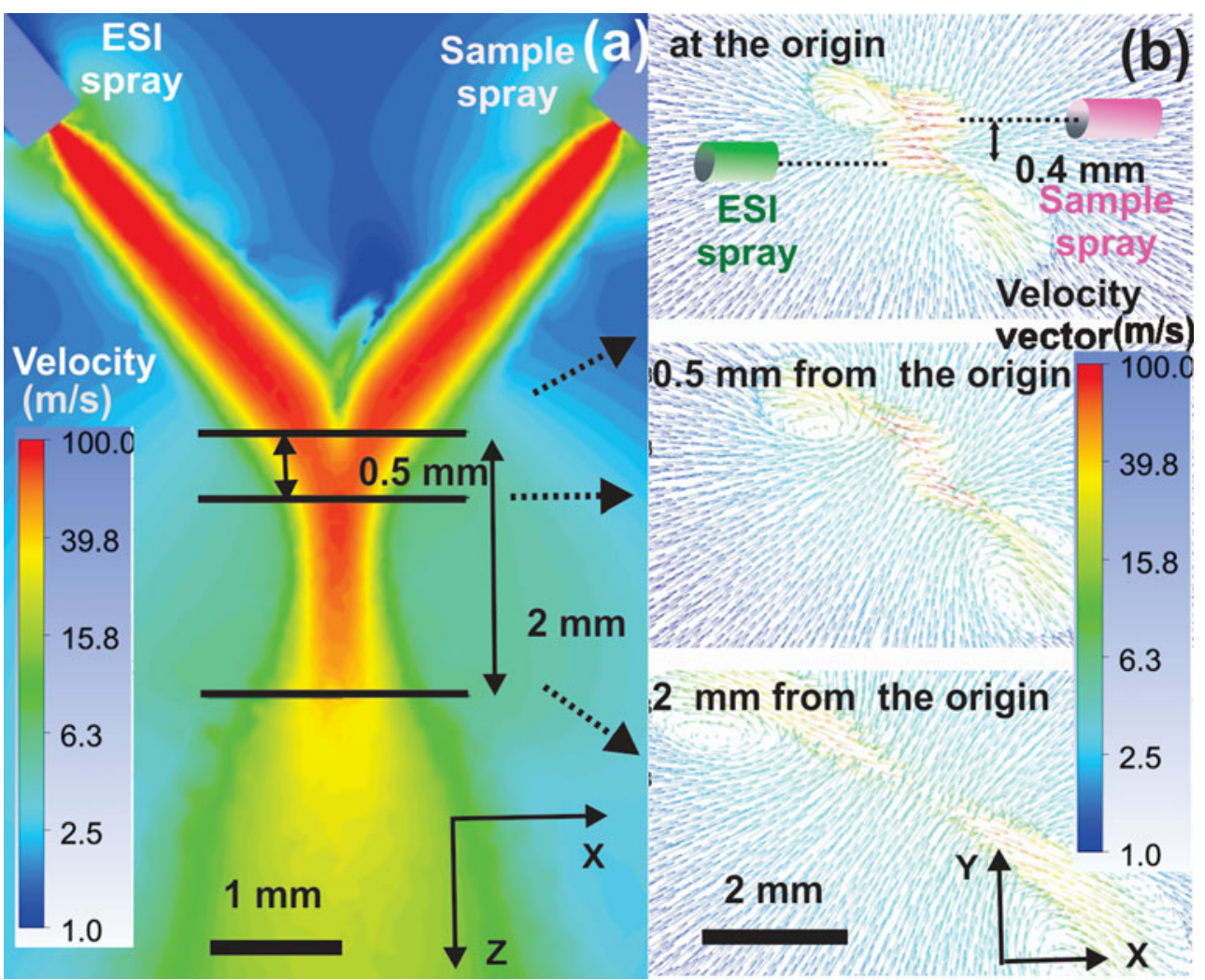

the directions of $V_{\mathrm{E}}$ and $V_{\mathrm{S}}$. We is the ratio of the kinetic energy (it is the CKE in the droplet-droplet collision) to the surface energy of droplets defined as:

$W e=\rho U^{2} D_{\mathrm{E}} / \sigma$

$D_{\mathrm{E}}$ is the diameter of an ESI droplet. The diameter of the smaller droplet is used to calculate We. $D_{\mathrm{E}}$ is used here because in the EESI process, the ESI droplets are usually smaller than the neutral sample droplets. $\rho$ and $\sigma$ are the density and surface tension of the solvents in the colliding droplets, respectively (the solvents in two droplets are assumed to be the same in Eq. 2). Combining Eqs. 1 and 2, We can be calculated as follows:

$W e=\rho\left(V_{\mathrm{E}}^{2}+V_{\mathrm{S}}^{2}-2 V_{E} V_{S} \cos \theta\right) D_{E} / \sigma$

For $D_{\mathrm{E}} V_{\mathrm{E}}$, and $V_{\mathrm{S}}$, we use the average diameter of the ESI droplets and the average velocities of the ESI and sample droplets from the whole measurement area covered by the PDA experiments. These values are $5.09 \mu \mathrm{m}$ for $D_{\mathrm{E}}$, $39.67 \mathrm{~m} / \mathrm{s}$ for $V_{E}$, and $21.04 \mathrm{~m} / \mathrm{s}$ for $V_{\mathrm{S}}$. For simplicity, the volume ratios of water to ethanol in the two droplets are assumed to be the same as the ones in the bulk solution, i.e., solvent evaporation and vapor condensation are ignored. The density and surface tension of a 1:1 water/ethanol mixture are $0.9260 \mathrm{~g} / \mathrm{cm}^{3}\left(20{ }^{\circ} \mathrm{C}\right)[29]$ and $30.69 \mathrm{~m} \mathrm{Nm}^{-1}\left(20{ }^{\circ} \mathrm{C}\right)[30]$. Considering a variation of $\theta$ from $0^{\circ}$ to $180^{\circ}$, the value of $W e$ is 53.30 at $\theta=0^{\circ}$ and 566.05 at $\theta=180^{\circ}$, i.e., We covers a range of more than an order of magnitude. An increase of We means an increase in the ratio of kinetic energy to surface energy according to the definition of $W e$. When the value of $W e$ is lower than 10 , bounce usually occurs in dropletdroplet collisions [31, 32] because the kinetic energy is insufficient to overcome the surface energy and to expel the intervening gas film. When the value of $W e$ is too high, e. g., 100, two droplets will coalesce temporarily and the excess kinetic energy can be transferred to rotational energy, which causes the coalesced droplet to separate, resulting in fragmentation or disruption. Gunn [32] concluded that the minimum kinetic energy to expel the intervening gas film is exceeded when the relative velocity of the droplets $U$ is greater than a critical value, $\sqrt{8 \sigma / \rho D_{\mathrm{S}}}$. Here, $D_{\mathrm{S}}$ is the diameter of the sample droplet, which is the larger droplet used to calculate the critical value of $U$. The average diameter of the sample droplets is used as $D_{\mathrm{S}}(12.32 \mu \mathrm{m})$. In the EESI process, the critical value is estimated to be $4.64 \mathrm{~m} / \mathrm{s}$, much smaller than the actual value of $U$, which is in the range from 18.63 to $60.71 \mathrm{~m} / \mathrm{s}$ depending on $\theta\left(0-180^{\circ}\right)$. This clearly demonstrates that bounce is not the dominant process following droplet-droplet collision in EESI. Furthermore, different from disruption, fragmentation generates satellite droplets [17]. The PDA measurements showed the slightly smaller value of the SMD in the EESI spray compared with the average SMD at the origin. This suggests the production of 
satellite droplets at the origin, which are too small to be detected by PDA $(<1 \mu \mathrm{m})$. Therefore, fragmentation may be the dominant process following droplet-droplet collision in an EESI plume, which is considered to be beneficial for EESIMS measurements due to the production of small satellite droplets with better desolvation characteristics and higher charge densities.

Previous studies have indicated that for a larger diameter ratio of two droplets, there is a larger region of $b$ for stable coalescence when the value of We is fixed [33, 34]. In the EESI process, the sizes of the droplets in the ESI spray and in the sample spray are not dramatically different, implying that the chance of total coalescence is low in EESI, consistent with the results obtained in the PDA measurements. In addition, when the impact parameter $b$ is large, but still smaller than $1 / 2\left(D_{\mathrm{S}}+D_{\mathrm{E}}\right)$, droplets may collide with temporary contact, followed by "catastrophic" breakup. In such a case, the fluid transfer direction between two droplets influences the material transfer during the collision. For colliding droplets with unequal size, the fluid from the smaller droplet is known to flow to the larger droplet as soon as contact has been made [17]. PDA measurements show that ESI droplets are usually smaller than sample droplets, implying that it is more likely for the fluid from the ESI droplet to flow toward the sample droplet. In this case, if the sample contains salts, buffers, or matrices at high concentrations, these compounds could not be efficiently separated from the analytes due to the fluid transfer direction. To optimize EESI in terms of matrix

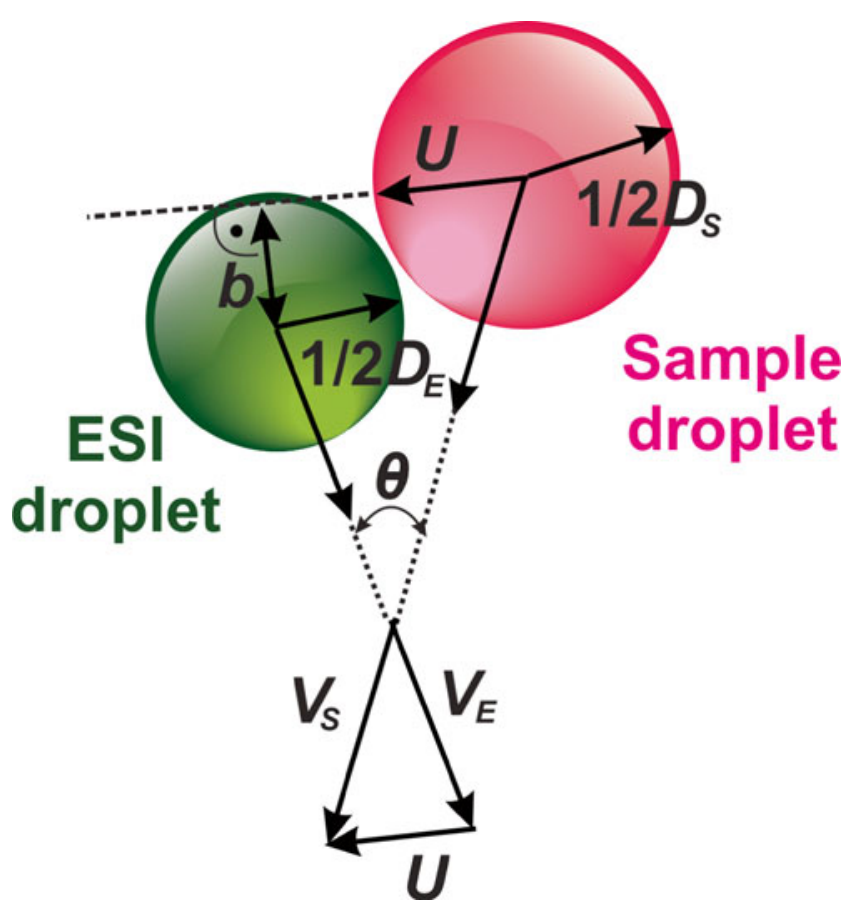

Fig. 7 Schematic of droplet-droplet collision tolerance, it would be advantageous to have matrix from the sample droplets being diluted by flowing into the ESI droplets during a transient contact. Hence, the sample droplets should be slightly smaller than the size of the ESI droplets. This is hard to achieve purely with the help of a sheath gas. Additional methods are needed, such as adjustment of the capillary size to reduce the droplet size, or an ultrasonic transducer [12] or an aerosol generator [35] to deliver the sample via a plume of fine droplets $(\leq 1 \mu \mathrm{m})$.

Besides the issue of fluid transfer between the ESI droplets and the sample droplets, other factors are also crucial for enhancing ion signals in EESI-MS measurements, including the lifetime of the temporary coalesced droplets and the collision probability between the ESI droplets and the sample droplets. The extraction of the analytes mostly happens during the period of temporary coalescence. In order to let more analytes be extracted to the charged solvents, the lifetime $t$ of temporary coalesced droplets should be as long as possible. $t$ can be estimated by the oscillation time of a natural droplet, as shown below $[36,37]$ :

$t=2 \pi\left(\rho R_{0}^{3} / 8 \sigma\right)^{1 / 2}$

where $R_{0}$ is the radius of the temporary coalesced droplet. $R_{0}$ can be estimated as $10 \mu \mathrm{m}$ from the experimental data, and $t$ is calculated to be $4.63 \mu \mathrm{s}$. It is possible to increase the value of $t$ by optimizing the solvent components or increasing the droplet size of the ESI spray and the sample spray. Furthermore, PDA measurements show that the overlap of the droplet distributions between the ESI spray and the sample spray at the origin and downstream from the origin was fairly small due to the gas flow effect. This definitely reduces the collision probability between the ESI droplets and the sample droplets. Therefore, an auxiliary setup could help confine the two sprays to increase the overlap area of the two droplet distributions; for example, a third gas flow together with a sealed chamber can be used to confine the two gas flows by producing turbulence to disturb the original gas flow pattern intentionally.

\section{Conclusions}

In this work, the mechanism of EESI in the dual-spray configuration has been clarified based on the systematic PDA measurements, numeric simulations, and theoretical analysis. Measurements of droplet size, velocity, and density were conducted in the EESI spray at the origin and downstream from the origin using various experimental parameters. The droplet behavior in the single-spray mode was also investigated to compare with the results in the EESI spray. The results show that the droplet size 
distribution of the EESI spray was very similar to the averaged results in single-spray mode. This suggests that there was no permanent coalescence at the origin as well as further downstream in the EESI plume, although the solvents in the two sprays were identical. Similar phenomena were observed with different geometries of the EESI source and various sample flow rates. The absence of coalescence probably has two reasons: (1) the overlap of the droplet distributions between the ESI spray and the sample spray was small, reducing the collision probability between the ESI droplets and sample droplets. (2) The kinetic energy of the droplet collisions in the EESI process was too high to produce stable coalesced droplets. Moreover, PDA measurements and numerical simulations indicate that the two sprays were offcenter in the experiments. The results of droplet size measurements suggest that fragmentation rather than disruption may be the dominant type of droplet-droplet interaction following collision. Theoretical analysis suggests that increasing the size of ESI droplets compared with the size of sample droplets would enhance the chance of fragmentation and reduce the matrix effect on the analytes. This systematic work provides a comprehensive view of the mechanism of EESI process and allows practical suggestions for the optimization of EESI.

Acknowledgments We would like to thank Mr. Lukas Meier, Dr. Thomas Schmid, and Dr. Waisiang Law for their kind help and nice discussion. This project was supported by the Swiss National Science Foundation (grant no. 200020-124663).

\section{References}

1. Chen HW, Venter A, Cooks RG (2006) Extractive electrospray ionization for direct analysis of undiluted urine, milk and other complex mixtures without sample preparation. Chem Commun 19:2042-2044

2. Marquez CA, Wang HY, Fabbretti F, Metzger JO (2008) Electrontransfer-catalyzed dimerization of trans-anethole: detection of the distonic tetramethylene radical cation intermediate by extractive electrospray iIonization mass spectrometry. J Am Chem Soc 130 (51):17208-17209

3. Chen HW, Yang SP, Li M, Hu B, Li JQ, Wang J (2010) Sensitive detection of native proteins using extractive electrospray ionization mass spectrometry. Angew Chem-Int Edit 49(17):3053-3056

4. Luo MB, Hu B, Zhang X, Peng DF, Chen HW, Zhang LL, Huan YF (2010) Extractive electrospray ionization mass spectrometry for sensitive detection of uranyl species in natural water samples. Anal Chem 82(1):282-289

5. Gu HW, Chen HW, Pan ZZ, Jackson AU, Talaty N, Xi BW, Kissinger C, Duda C, Mann D, Raftery D, Cooks RG (2007) Monitoring diet effects via biofluids and their implications for metabolomics studies. Anal Chem 79(1):89-97

6. Miao ZX, Chen H (2009) Direct analysis of liquid samples by desorption electrospray ionization-mass spectrometry (DESI-MS). J Am Soc Mass Spectrom 20(1):10-19

7. Iribarne JV, Thomson BA (1976) On the evaporation of small ions from charged droplets. J Chem Phys 64(6):2287-2294

8. Dole M, Mack LL, Hines RL (1968) Molecular beams of macroions. J Chem Phys 49(5):2240-2249
9. Law WS, Wang R, Hu B, Berchtold C, Meier L, Chen HW, Zenobi R (2010) On the mechanism of extractive electrospray ionization. Anal Chem 82(11):4494-4500

10. Ding JH, Yang SP, Liang DP, Chen HW, Wu ZZ, Zhang LL, Ren YL (2009) Development of extractive electrospray ionization ion trap mass spectrometry for in vivo breath analysis. Analyst 134 (10):2040-2050

11. Gamez G, Zhu LA, Disko A, Chen HW, Azov V, Chingin K, Kramer G, Zenobi R (2011) Real-time, in vivo monitoring and pharmacokinetics of valproic acid via a novel biomarker in exhaled breath. Chem Commun 47(17):4884-4886

12. Zhu L, Gamez G, Chen HW, Chingin K, Zenobi R (2009) Rapid detection of melamine in untreated milk and wheat gluten by ultrasound-assisted extractive electrospray ionization mass spectrometry (EESI-MS). Chem Commun 5:559-561

13. Yang SP, Ding JH, Zheng J, Hu B, Li JQ, Chen HW, Zhou ZQ, Qiao XL (2009) Detection of melamine in milk products by surface desorption atmospheric pressure chemical ionization mass spectrometry. Anal Chem 81(7):2426-2436

14. Law WS, Chen HW, Ding JH, Yang SP, Zhu L, Gamez G, Chingin K, Ren YL, Zenobi R (2009) Rapid characterization of complex viscous liquids at the molecular level. Angew Chem-Int Edit 48(44):8277-8280

15. Ding JH, Gu HW, Yang SP, Li M, Li JQ, Chen HW (2009) Selective detection of eiethylene glycol in toothpaste products using neutral desorption reactive extractive electrospray ionization tandem mass spectrometry. Anal Chem 81(20):8632-8638

16. Law WS, Chen HW, Balabin R, Berchtold C, Meier L, Zenobi R (2010) Rapid fingerprinting and classification of extra virgin olive oil by microjet sampling and extractive electrospray ionization mass spectrometry. Analyst 135(4):773-778

17. Orme M (1997) Experiments on droplet collisions, bounce, coalescence and disruption. Prog Energy Combust Sci 23(1):65-79

18. Gao TC, Chen RH, Pu JY, Lin TH (2005) Collision between an ethanol drop and a water drop. Exp Fluids 38(6):731-738

19. Gomez A, Tang KQ (1994) Charge and fission of droplets in electrostatic sprays. Phys Fluids 6(1):404-414

20. Tang K, Gomez A (1994) On the structure of an electrostatic spray of monodisperse droplets. Phys Fluids 6(7):2317-2332

21. Olumee Z, Callahan JH, Vertes A (1998) Droplet dynamics changes in electrostatic sprays of methanol-water mixtures. J Phys Chem A 102(46):9154-9160

22. Venter A, Sojka PE, Cooks RG (2006) Droplet dynamics and ionization mechanisms in desorption electrospray ionization mass spectrometry. Anal Chem 78(24):8549-8555

23. Heine MC (2007) Particle dynamics at high aerosol concentrations and production rates. ETHZ, Zurich

24. Heine MC, Pratsinis SE (2005) Droplet and particle dynamics during flame spray synthesis of nanoparticles. Ind Eng Chem Res 44(16):6222-6232

25. Hindes WC (1999) Aerosol technology: properties, behavior, and measurement of airborne particles. Wiley, New York

26. Hardalupas Y, Liu CH, Whitelaw JH (1994) Experiments with disk stabilized kerosene-fuelled flames. Combust Sci Technol 97 (1-3):157-191

27. Issac K, Missoum A, Drallmeier J, Johnston A (1994) Atomization experiments in a coaxial coflowing Mach 1.5 flow. AIAA J 32 (8):1640-1646

28. Mackay GDM, Mason SG (1963) The gravity approach and coalescence of fluid drops at liquid interfaces. CanJChemEng 41:203-212

29. Dizechi M, Marschall E (1982) Viscosity of some binary and ternary liquid mixtures. J Chem Eng Data 27(3):358-363

30. Vazquez G, Alvarez E, Navaza JM (1995) Surface tension of alcohol + water from 20 to $50^{\circ} \mathrm{C}$. J Chem Eng Data 40(3):611614 
31. Cheng CS, Li YQ, Zhao LF (2009) Investigation of improving the model for binary collision regimes of hydrocarbon droplets. In: Wenbin H, Xin L (eds) 2009 International Conference on Information Engineering and Computer Science, Wuhan, China, 19-20 December 2009, IEEE

32. Gunn R (1965) Collision characteristics of freely falling water drops. Science 150(3697):695-701

33. Low TB, List R (1982) Collision, coalescence and breakup of raindrops. Part I: Experimentally established coalescence efficiencies and fragment size distributions in breakup. J Atmos Sci 39(7):15911606
34. Braziers PR, Latham J, Jennings SG (1972) The interaction of falling water drops: coalescence. Proc R Soc Lond A 326 (1566):393-408

35. Gu HW, Hu B, Li JQ, Yang SP, Han J, Chen HW (2010) Rapid analysis of aerosol drugs using nano extractive electrospray ionization tandem mass spectrometry. Analyst 135(6):12591267

36. Peng Z, Law CK (2011) An analysis of head-on droplet collision with large deformation in gaseous medium. Phys Fluids 23 (4):042102

37. Lamb H (ed) (1932) Hydrodynamics, 6th edn. Dover, New York 